\title{
Cardiac surgeon wanted: mouse experience required?
}

$\mathrm{H}$ arry Schwartz, M.D. was a skilled cardiac surgeon with many years of experience performing heart transplants, valve replacements, and other technically challenging procedures at the Great Eastern University hospital. Schwartz also had a long history of using dogs and swine for cardiac research and teaching. Therefore, the members of the Great Eastern IACUC raised no objections when Dr. Amos White listed Schwartz as the cardiac surgeon on his IACUC protocol. The protocol only required Schwartz to ligate the main cardiac arteries of adult mice. The animals were meant to recover from the procedure.

Unfortunately, all five of the mice operated on during the first day of surgery died before any of the school's veterinarians were advised of the problem. Later that day a veterinarian heard of the deaths and questioned Schwartz. Schwartz said that it was reasonable for some mortality to occur at first because his surgical technique was being perfected. Nevertheless, the veterinarian told Schwartz to immediately stop his participation on the protocol until the IACUC could review the circumstances surrounding the animals' deaths.

When questioned by the IACUC, Schwartz said that he had truthfully answered all the questions on the IACUC application form. Specifically, he had responded that he was a board-certified surgeon with over 30 years of experience with cardiac surgery and he had operated on animal hearts on many previous occasions. However, the protocol application never specifically questioned if he had performed on mice the cardiac procedure he was to do for White, so he never addressed that subject. Although the IACUC could not understand how Schwartz could blatantly misrepresent his expertise, Schwartz argued that there was no misrepresentation at all and that the fault was with the IACUC for not asking more specific questions on the application form. He told the committee that the deaths had occurred before the mice had emerged from anesthesia, and that now he was confident that he had perfected the skills needed to perform the technique and he was ready to proceed with the protocol.

What are the next steps to be taken by the IACUC?

\section{Jerald Silverman \\ University of Massachusetts Medical School, Worcester, MA, USA \\ e-mail: Jerald.Silverman@umassmed.edu}

Published online: 23 October 2019

https://doi.org/10.1038/s41684-019-0417-4

\section{Focus on the future: training is better than blaming}

T his is not an uncommon scenario and, as with any real-life situation involving multiple parties, the decision as to what to do next is a complicated one. Here we have a highly trained, highly experienced physician who has worked with many different species, including humans. To the lab, therefore, he was a logical choice to add to the research team when they needed a cardiac surgeon, and the IACUC of Great Eastern University (GEU) amenably approved this modification. After all, he was "only" needed to ligate a few cardiac arteries in some mice. However, it turned out that despite his vast training in other species, the surgeon did not have experience with the proposed survival procedure in the species in question, and the first five animals died soon after surgery.

The question posed in this scenario is, quite appropriately, "what should the IACUC do next," rather than "who is to blame." It does no good (especially for the animals) to squabble over who intentionally or unintentionally misled whom; instead, this can be used as an opportunity for collaboration between the IACUC, the veterinary staff, and the lab. The most immediate concern is the welfare of the animals, so the veterinarians did the right thing in provisionally preventing the surgeon from performing surgeries on mice. It should now be made clear to both him and the PI (if it has not already) that surgeries cannot proceed until the IACUC and veterinarians are satisfied that, indeed, the "skills needed to perform" them have been "perfected."

Given their mandated role to consider the "adequacy of training and experience of personnel in the procedures used"1-3, the IACUC then needs to clearly assess the surgeon's background with mouse cardiac surgery. This may require IACUC-specific training (e.g., mouse handling and aseptic technique modules in CITI or the AALAS Learning Library) but should also include a request for the surgeon's own reported background with this and similar work. Most importantly, perhaps, the IACUC should require that a veterinarian either oversee the next set of surgeries on a limited number of animals (preferred) or receive a report from the PI after these procedures are performed.

Once the IACUC is satisfied that these surgical procedures can be performed appropriately, some form of post-approval monitoring would be prudent, either as a formal follow-up a few weeks/months later or as part of the next round of semiannual inspections. Either way, the IACUC should also consider how information regarding training/experience is initially acquired and assessed by the Committee and whether further clarification on animal use protocol applications is necessary. These steps should help to ensure the welfare of the animals while fostering meaningful collaboration between the IACUC and the research community in order to create a positive and safe working environment for all.

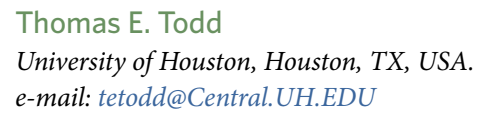

Published online: 23 October 2019 https://doi.org/10.1038/s41684-019-0420-9

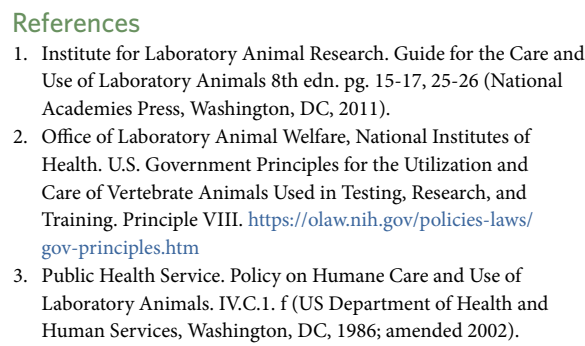

